\title{
LIOUVILLIAN FIRST INTEGRALS FOR QUADRATIC SYSTEMS WITH AN INTEGRABLE SADDLE
}

\author{
YUDY BOLAÑOS, JAUME LLIBRE AND CLAUDIA VALLS
}

ABSTRACT. We provide explicit expressions for the Liouvillian first integrals of the quadratic polynomial differential systems having an integrable saddle.

1. Introduction. Let $\mathbb{R}[x, y]$ be the ring of all polynomials in the variables $x$ and $y$ and with coefficients in $\mathbb{R}$.

A quadratic polynomial differential system or simply a quadratic system is a polynomial differential system in $\mathbb{R}^{2}$ of the form

$$
\dot{x}=P(x, y), \quad \dot{y}=Q(x, y),
$$

where $P, Q \in \mathbb{R}[x, y]$ and the maximum of the degrees of $P$ and $Q$ is 2 .

Quadratic differential systems have been widely studied in the last 100 years, and more than 1,000 papers have been published about them (see, for instance, $[\mathbf{1 2}, \mathbf{1 6}, \mathbf{1 7}]$ ). These systems are considered as one of the easiest, but not trivial, families of nonlinear differential systems, although the problem of classifying all quadratic vector fields (even integrable ones) still remains open. For more information on the integrable differential vector fields in dimension 2 , see for instance, [3]).

The classification of the centers for the quadratic systems has a long history which started with the works of Dulac [5], Kapteyn [9, 10], Bautin [2], Zoladek [18], etc. Schlomiuk, Guckenheimer and Rand in [13, pages 3, 4 and 13] described a brief history of the problem of the

2010 AMS Mathematics subject classification. Primary 34A34, 34C05, 34C14.

Keywords and phrases. quadratic systems, integrable saddle, Liouvillian first integral, integrating factor, inverse integrating factor.

The second author is supported by grants MICINN/FEDER MTM 2008-03437, Generalitat de Catalunya 2009SGR410, ICREA Academia and FP7-PEOPLE-2012IRSES-316338. The third author is supported by grants AGAUR PIV-DGR-2010 and by the FCT through the project PTDC/MAT/ 117106/2010 and through CAMGD, Lisbon.

Received by the editors on December 29, 2012, and in revised form on January 21, 2014. 
center in general, and it includes a list of 30 papers covering the topic and the turbulent history of the center for the quadratic case.

The weak focus and the quadratic centers are classified using the Lyapunov constants $V_{1}, V_{2}$ and $V_{3}$. Dulac [5] was the first to detect that the weak focus and the quadratic centers can pass to weak saddles and integrable saddles through a complex change of variables, see for details, [8]. Recently, such kinds of saddles have been studied by several authors Sulin [15], Joyal and Rousseau [8], and Artés, Llibre and Vulpe [1]. These last authors characterized the phase portraits of all quadratic systems having an integrable saddle, but they did not provide their first integrals. This will be the main objective of this paper.

The polynomial differential system (1.1) is integrable on an open and dense subset $U$ of $\mathbb{R}^{2}$ if there exists a non-constant $\mathcal{C}^{1}$ function $H: U \rightarrow \mathbb{R}$, called a first integral of the system on $U$, which is constant on all solution curves $(x(t), y(t))$ of system (1.1) contained in $U$, i.e., $H(x(t), y(t))$ is constant for all values of $t$ for which the solution $(x(t), y(t))$ is defined and contained in $U$, or, in other words,

$$
P \frac{\partial H}{\partial x}+Q \frac{\partial H}{\partial y}=0
$$

for the points of $U$.

Let $W$ be a simple, connected open and dense subset of $\mathbb{R}^{2}$. A nonzero $\mathcal{C}^{1}$ function $V: W \rightarrow \mathbb{R}$ is an inverse integrating factor of system (1.1) on $W$ if it is a solution of linear partial differential equation

$$
P \frac{\partial V}{\partial x}+Q \frac{\partial V}{\partial y}=\operatorname{div}(P, Q) V
$$

where $\operatorname{div}(P, Q)=\partial P / \partial x+\partial Q / \partial y$ denotes the divergence of vector field $\mathcal{X}=(P, Q)$ associated to system (1.1).

A weak saddle is a hyperbolic saddle such that the trace of its linear part is zero. More precisely, from $[\mathbf{1}, \mathbf{5}, \mathbf{8}, \mathbf{1 5}]$, if a quadratic system possesses a weak saddle via an affine transformation, this system can be written as

$$
\begin{aligned}
& \dot{x}=x+a x^{2}+b x y+c y^{2}, \\
& \dot{y}=-y-k x^{2}-l x y-m y^{2},
\end{aligned}
$$


with the weak saddle at the origin. Moreover, we say that the origin is an integrable saddle if

$$
\begin{aligned}
& L_{1}=l m-a b=0, \\
& L_{2}=k b(2 m-b)(m+2 b)-c l(2 a-l)(a+2 l)=0, \\
& L_{3}=(c k-l b)[\operatorname{acl}(2 a-l)-b k m(2 m-b)]=0 .
\end{aligned}
$$

Taking into account these conditions it is obtained in [1] that the quadratic systems with an integrable saddle can be reduced to the following five families of quadratic systems:

$$
\begin{gathered}
\dot{x}=x-2 c k x^{2}+x y+c y^{2}, \\
\dot{y}=-y-k x^{2}-c k x y+2 y^{2}, \\
\dot{x}=x+m x^{2}+x y+c y^{2}, \\
\dot{y}=-y-c x^{2}-x y-m y^{2}, \\
\dot{x}=x+l m x^{2}+x y+c y^{2}, \\
\dot{y}=-y-c l^{3} x^{2}-l x y-m y^{2}, \\
\quad \dot{x}=x+a x^{2}+c y^{2}, \\
\quad \dot{y}=-y-k x^{2}-m y^{2}, \\
\dot{x}=x+a x^{2}+2 m x y+c y^{2}, \\
\dot{y}=-y-k x^{2}-2 a x y-m y^{2} .
\end{gathered}
$$

It is known that all quadratic systems with an integrable saddle possess a Liouvillian first integral, see for instance, [1], or the appendix where we explain how it is known that all integrable saddles have a Liouvillian first integral. We recall that a Liouvillian first integral is a first integral that can be expressed by quadratures of elementary functions, see for more details, [14]. This is the reason for calling the weak saddles satisfying $L_{1}=L_{2}=L_{3}=0$, integrable saddles. The objective of this paper is to provide the explicit expressions of these first integrals for each of the families (1.3)-(1.7). 
2. Statement of the main results. We need to recall that a polynomial differential system (1.1) with an inverse integrating factor $V=V(x, y): W \rightarrow \mathbb{R}$ and a first integral $H$ associated to $V$ satisfies

$$
\dot{x}=\frac{P}{V}=\frac{\partial H}{\partial y}, \quad \dot{y}=\frac{Q}{V}=-\frac{\partial H}{\partial x} .
$$

Therefore,

$$
H(x, y)=\int \frac{P(x, y)}{V(x, y)} d y+g(x) .
$$

We note that the function $g(x)$ depends only on $x$ because it is a constant of integration with respect to the variable $y$. Moreover, $g(x)$ can be computed from the equation

$$
\frac{\partial H}{\partial x}=-\frac{Q}{V} .
$$

Theorem 2.1. The quadratic systems (1.3)-(1.7) possess a polynomial inverse integrating factor $V=V(x, y)$.

(a) For system (1.3), $V=V_{11} V_{12}$ with

$$
\begin{aligned}
V_{11}= & k x^{2}-2 c k x y+c^{2} k y^{2}+2 c k x+2 y-1, \\
V_{12}= & \left(1-c^{2} k\right)\left(k x^{3}-3 c k x^{2} y+3 c^{2} k x y^{2}-c^{3} k y^{3}+3 c k x^{2}\right. \\
& \left.+3\left(1-c^{2} k\right) x y-3 c y^{2}\right)+6 c(c k x+y)-2 c .
\end{aligned}
$$

(b) For system (1.4), $V=V_{21} V_{22}$ with

$$
\begin{aligned}
V_{21}= & (c-m)(x+y)-1, \\
V_{22}= & (2 c-1)(c+m-1)\left(c x^{2}-(c-m-1) x y+c y^{2}\right) \\
& +2 c((c+m-1)(x+y)+1) .
\end{aligned}
$$

(c) For system (1.5), $V=V_{31} V_{32}$ with

$$
\begin{aligned}
V_{31} & =1-(c l-m)(l x+y), \\
V_{32} & =2 c-(2 c l-1)\left((c l-1)^{2}-m^{2}\right) x y \\
& +2 c(c l+m-1)(l x+y)+c(2 c l-1)(c l+m-1)\left(l^{2} x^{2}+y^{2}\right) .
\end{aligned}
$$


(d) For system (1.6)

$$
\begin{aligned}
V= & (c k-a m)\left(k x^{3}+3 x y+a x^{2} y+m x y^{2}+c y^{3}\right) \\
& -\left(a^{2}+k m\right) x^{2}-\left(a c+m^{2}\right) y^{2}-2 a x-2 m y-1 .
\end{aligned}
$$

(e) For system (1.7) $V=1$. So, system (1.7) is Hamiltonian.

In 1992, Singer [14] proved that a polynomial differential system has a Liouvillian first integral if and only if it has an inverse integrating factor of the form

$$
\exp \left(\int U_{1}(x, y) d x+\int U_{2}(x, y) d y\right),
$$

where $U_{1}$ and $U_{2}$ are rational functions which verify $\partial U_{1} / \partial y=\partial U_{2} / \partial x$. In 1999, Christopher [4] improved the results of Singer showing that the inverse integrating factor (2.2) can be written in the form

$$
\exp (g / h) \prod_{i=1}^{k} f_{i}^{\lambda_{i}}
$$

where $g, h$ and $f_{i}$ are polynomials and $\lambda_{i} \in \mathbb{C}$.

Since all inverse integrating factors of Theorem 2.1 are polynomial, they are of the form (2.3). Consequently, by the results of Singer and Christopher we have given a new proof that all the first integrals of systems (1.3)-(1.7) are Liouvillian. Now we shall give the explicit expressions of these first integrals.

Theorem 2.2. The following statements hold.

(a) A first integral of system (1.3) is

$$
\begin{array}{ll}
\frac{V_{11}^{3}}{V_{12}^{2}} & \text { if } 1+c^{2} k \neq 0, \\
\frac{4 c x+6 x^{2}+c^{2}\left(1-4 y+6 y^{2}\right)}{V_{11}^{2}} & \text { if } 1+c^{2} k=0 .
\end{array}
$$

(b) A first integral of system (1.4) is:

$$
\begin{array}{ll}
V_{21}^{c+m-1} V_{22}^{c-m} & \text { if } m \neq c, 1-c, 3 c-1, c \neq 1 / 2, \\
(2 c-1)(x+y)-\log \left|V_{22}\right| & \text { if } m=c \neq 1 / 2,
\end{array}
$$




$$
\begin{aligned}
& (2 c-1)\left(c(2 c-1)\left(x^{2}+y^{2}\right)\right. \\
& \left.+\left(-4 c^{2}+6 c-2\right) x y+2 c(x+y)\right) \\
& +2 c \log \left|V_{21}\right| \quad \text { if } m=1-c \neq 1 / 2, \\
& \frac{1}{V_{21}^{2}}\left(2(2 c-1)\left(2 c+(1-2 c)^{2} x\right) y+c(3+4(2 c-1) x)\right) \\
& \quad+2 c \log \left|V_{21}\right| \quad \text { if } m=3 c-1 \neq 1 / 2, \\
& \frac{1}{V_{22}}\left(4 V_{22}-8+(2 m-1)^{2}\left(x^{2}+(2 m+1) x y+y^{2}\right)\right. \\
& \left.-8 V_{22} \log \left|2 V_{22}\right|\right) \quad \text { if } c=1 / 2, m \neq 1 / 2 ;
\end{aligned}
$$

moreover, if $m=c=1 / 2$, system (1.4) coincides with system (1.7) with $a=k=1 / 2$.

(c) A first integral of system (1.5) is:

$$
\begin{aligned}
& \frac{V_{32}^{m-c l}}{V_{31}^{m+c l-1}} \\
& \text { if } m \neq c l, 1-c l, 3 c l-1,2 c l \neq 1 \text {, } \\
& \frac{1}{V_{32}}\left(4 c\left(2 V_{32}-8 c\right)+(2 m-1)^{2}\left(x^{2}+2 c(2 m+1) x y+4 c^{2} y^{2}\right)\right. \\
& \left.-16 c V_{32} \log \left|2 V_{32}\right|\right) \quad \text { if } 2 c l=1, m \neq 1 / 2, \\
& (2 c l-1)(l x+y)-\log \left|V_{32}\right| \quad \text { if } m=c l, 2 c l \neq 1 \text {, } \\
& (2 c l-1)((-2+2 c l(3-2 c l)) x y \\
& \left.+2 c(l x+y)+c(2 c l-1)\left(l^{2} x^{2}+y^{2}\right)\right) \\
& 2 c \log \left|V_{31}\right| \quad \text { if } m=1-c l, 2 c l \neq 1 \text {, } \\
& \frac{1}{V_{31}^{2}}\left[-16 c^{3} l^{4} x^{2}+2 x(l x-1)\right. \\
& \left.+8 c^{2} l^{2} x\left(3 l x-1+c\left(-12 l^{2} x^{2}+8 l x-1\right)\right)\right] \\
& +\frac{2}{V_{31}}\left(x+4 c^{2} l^{2} x+c(2-4 l x)\right) \\
& +2 c \log \left|V_{31}\right| \quad \text { if } m=3 c l-1,2 c l \neq 1 \text {; }
\end{aligned}
$$

moreover, if $m=1 / 2$ and $2 c l=1$, system (1.5) coincides with system (1.7) with $a=1 /(4 c)$ and $k=1 /\left(8 c^{2}\right)$.

(d) A first integral of system (1.6) is

$$
\sum_{i=1}^{3} \frac{\left(x+a x^{2}+c r_{i}^{2}\right) \log \left|y-r_{i}\right|}{f\left(r_{i}\right)}
$$


where $f(r)=3 c(a m-c k) r^{2}+2\left(a c+m^{2}-c k m x+a m^{2} x\right) r+a(a m-$ $c k) x^{2}+3(a m-c k) x+2 m$ and $r_{1}, r_{2}$ and $r_{3}$ are the three roots of the following polynomial in the variable $r$ :

$$
\begin{aligned}
\left(-c^{2} k+a c m\right) r^{3}+ & \left(a c+m^{2}-c k m x+a m^{2} x\right) r^{2} \\
+(2 m- & \left.3 c k x+3 a m x-a c k x^{2}+a^{2} m x^{2}\right) r \\
& +\left(2 a x+a^{2} x^{2}+k m x^{2}-c k^{2} x^{3}+a k m x^{3}+1\right) .
\end{aligned}
$$

(e) A first integral of Hamiltonian system (1.7) is

$$
k x^{3}+3 a x^{2} y+3 m x y^{2}+c y^{3}+3 x y .
$$

\section{Proof of Theorems 2.1 and 2.2 .}

Proof of Theorem 2.1. For each of the statements of the theorem, the formula for $V$ is obtained by looking for a polynomial solution of the linear partial differential equation (1.2).

For quadratic system (1.3), the equation (1.2) is

$$
\begin{aligned}
(x & \left.-2 c k x^{2}+x y+c y^{2}\right) \frac{\partial V}{\partial x} \\
& +\left(-y-k x^{2}-c k x y+2 y^{2}\right) \frac{\partial V}{\partial y}-(5(y-c k x)) V=0 .
\end{aligned}
$$

Once we look for a polynomial solution of (3.1) given by $V=V(x, y)$ of degree 5 , we get

$$
\begin{aligned}
V= & -\frac{1}{5 c^{4} k^{2}\left(c^{2} k-1\right)}\left(k x^{2}-2 c k x y+c^{2} k y^{2}+2 c k x+2 y-1\right) \\
& {\left[( 1 - c ^ { 2 } k ) \left(k x^{3}-3 c k x^{2} y+3 c^{2} k x y^{2}-c^{3} k y^{3}\right.\right.} \\
& \left.\left.+3 c k x^{2}+3\left(1-c^{2} k\right) x y-3 c y^{2}\right)+6 c(c k x+y)-2 c\right] .
\end{aligned}
$$

So this $V$ is an inverse integrating factor of system (1.3). Therefore, this proves statement (a) of the theorem.

Proceeding in a similar way, we obtain the inverse polynomial integrating factors of the quadratic systems (1.4)-(1.6). For quadratic system (1.7), we have $\operatorname{div}(P, Q)=0$ and, therefore, the system is Hamiltonian and its inverse integrating factor is 1 . 
Proof of Theorem 2.2. Since system (1.3) has a polynomial inverse integrating factor $V$ given by Theorem 2.1 (a), the first integral associated to $V$, see equation $(2.1)$, is

$$
H(x, y)=\int \frac{x-2 c k x^{2}+x y+c y^{2}}{V(x, y)} d y+g(x),
$$

satisfying

$$
\frac{\partial H}{\partial x}=-\frac{-y-k x^{2}-c k x y+2 y^{2}}{V} .
$$

Hence, we obtain $g(x)=0$, and

$$
\begin{gathered}
H=\frac{1}{6\left(1+c^{2} k\right)^{2}}\left(3 \log \left|-1+k x^{2}+2 y+c^{2} k y^{2}+x(2 c k-2 c k y)\right|\right. \\
-2 \log \mid\left(1-c^{2} k\right)\left(k x^{3}-3 c k x^{2} y+3 c^{2} k x y^{2}-c^{3} k y^{3}\right. \\
\left.\left.\quad+3 c k x^{2}+3\left(1-c^{2} k\right) x y-3 c y^{2}\right)+6 c(c k x+y)-2 c \mid\right),
\end{gathered}
$$

if $1+c^{2} k \neq 0$. Ignoring the constant appearing in $H$ we can write the first integral of the form

$$
3 \log \left|V_{11}\right|-2 \log \left|V_{12}\right|=\log \left|\frac{V_{11}^{3}}{V_{12}^{2}}\right|,
$$

where $V_{11}$ and $V_{12}$ are the functions defined in Theorem 2.1 (a). Finally, applying the exponential function to the above expression, we get the rational first integral of the quadratic system (1.3), so statement $(a)$ if $1+c^{2} k \neq 0$ is proved.

If $1+c^{2} k=0$, quadratic system (1.3) becomes

$$
\dot{x}=x+\frac{2 x^{2}}{c}+x y+c y^{2}, \quad \dot{y}=-y+\frac{1}{c^{2}} x^{2}+\frac{1}{c} x y+2 y^{2} .
$$

Again, by Theorem 2.1 (a), $V_{11}=-(c+x-c y)^{2} / c^{2}$ and $V_{12}=$ $-2(c+x-c y)^{3} / c^{2}$. We calculate the first integral associated to $V=V_{11} V_{12}$ computing (2.1) for this system, which is

$$
\frac{c^{2}\left(4 c x+6 x^{2}+c^{2}\left(1-4 y+6 y^{2}\right)\right)}{24(c+x-c y)^{4}}=\frac{4 c x+6 x^{2}+c^{2}\left(1-4 y+6 y^{2}\right)}{24 c^{2} V_{11}^{2}}
$$

removing the constant in the denominator we prove the rest of statement (a) of the theorem. 
Furthermore, by Theorem 2.1 (b), we have the inverse polynomial integration factor $V$ of system (1.4), and a first integral associated a $V$ for this system is obtained computing (2.1). Thus, we have the first integral

$$
\begin{aligned}
H= & \frac{1}{(2 c-1)(c-m)(3 c-m-1)(c+m-1)}[(c+m-1) \\
& \log |(c-m)(x+y)-1|+(c-m) \log \mid(2 c-1)(c+m-1) \\
& \left.\quad\left(c x^{2}-(c-m-1) x y+c y^{2}\right)+2 c((c+m-1)(x+y)+1) \mid\right],
\end{aligned}
$$

whenever $m \neq c, 1-c, 3 c-1$ and $c \neq 1 / 2$. Removing the constant which appears in the denominator, we write the remaining expression as

$$
(c+m-1) \log \left|V_{21}\right|+(c-m) \log \left|V_{22}\right|=\log \left|V_{21}^{c+m-1} V_{22}^{c-m}\right|,
$$

where $V_{21}$ and $V_{22}$ are the functions defined in Theorem 2.1 (b), and hence we obtain the first integral of statement (b) of the theorem for all $m \neq c, 1-c, 3 c-1$ and $c \neq 1 / 2$.

Calculating the first integral associated to the inverse integrating factor provided by Theorem 2.1 (b), for each of the remaining cases, we find that, if $m=c, V_{21}=-1$ and $V_{22}=(2 c-1)^{2}\left(c x^{2}+x y+c y^{2}\right)+$ $2 c((2 c-1)(x+y)+1)$, a first integral of the system is

$$
-\frac{(2 c-1)(x+y)-\log \left|V_{22}\right|}{(2 c-1)^{3}},
$$

for all $c \neq 1 / 2$. Although we can ignore the constant that appears in the denominator, it is easy to verify that the remaining expression is constant at $c=1 / 2$; therefore, it would not be a first integral of the system for this value of $c$. So the function obtained is a first integral whenever $m=c \neq 1 / 2$ as stated in statement (b) of the theorem.

Now, if $m=1-c$, we have $V_{21}=(2 c-1)(x+y)-1$ and $V_{22}=2 c$, and a first integral is

$$
\begin{aligned}
\frac{1}{4 c(2 c-1)^{3}}\left(( 2 c - 1 ) \left(c(2 c-1)\left(x^{2}+y^{2}\right)\right.\right. & +\left(-4 c^{2}+6 c-2\right) x y \\
+ & \left.2 c(x+y))+2 c \log \left|V_{21}\right|\right) .
\end{aligned}
$$

We observe that this function is not defined at $c=0,1 / 2$; however, eliminating the multiplicative constant, the first integral obtained is 
defined and it is not constant at $c=0$, but it is a complex constant if $c=1 / 2$. So, this last function is a first integral of the system if $m=1-c \neq 1 / 2$; thus, statement (b) for this case is proved.

Considering $m=3 c-1$, we have $V_{21}=(1-2 c)(x+y)-1$, $V_{22}=2 c(1+(2 c-1)(x+y))^{2}$, and the first integral is

$$
\frac{1}{4 c(2 c-1)^{3}}\left[\frac{c(3+4(2 c-1) x)+2(2 c-1)\left(2 c+(2 c-1)^{2} x\right) y}{V_{21}^{2}}+2 c \log \left|V_{21}\right|\right]
$$

with $c \neq 0,1 / 2$. Here also, we remark that the function obtained removing the multiplicative constant in the previous expression is defined, and it is not constant at $c=0$, whereas, in $c=1 / 2$, it is constant,; therefore, this function is the first integral of the system given in statement (b) of the theorem in the case $m=3 c-1 \neq 1 / 2$.

If $c=1 / 2$, then $V_{22}=(m-1 / 2)(x+y)+1=-V_{21}$, and the first integral is

$$
\frac{\left(4 V_{22}+(2 m-1)^{2}\left(x^{2}+(2 m+1) x y+y^{2}\right)-8 V_{22} \log \left|2 V_{22}\right|-8\right)}{(2 m-1)^{3} V_{22}}
$$

for all $m \neq 1 / 2$. It is easy to verify that, eliminating the constant that appears in the denominator, the function

$$
\frac{\left(4 V_{22}+(2 m-1)^{2}\left(x^{2}+(2 m+1) x y+y^{2}\right)-8 V_{22} \log \left|2 V_{22}\right|-8\right)}{V_{22}}
$$

is constant if $m=1 / 2$. So the function (3.2) is a first integral whenever $c=1 / 2$ and $m \neq 1 / 2$, and so we have proved statement (b) of the theorem for this case.

If $m=c=1 / 2$, the system is

$$
\dot{x}=x+x^{2} / 2+x y+y^{2} / 2, \quad \dot{y}=-x^{2} / 2-y-x y-y^{2} / 2,
$$

with inverse integrating factor $V=1$, so system (1.4) in this case is Hamiltonian, and it belongs to family (1.7) with $a=c=m=k=1 / 2$. Thus, we have completed the proof of statement (b) of the theorem.

For the quadratic system (1.5) the first integral associated to its 
polynomial inverse integrating factor provided by Theorem 2.1 (c) is:

$$
\begin{gathered}
\frac{1}{(2 c l-1)(c l-m)(3 c l-m-1)(c l+m-1)}[(m-c l) \log \mid 2 c \\
-(2 c l-1)\left((c l-1)^{2}-m^{2}\right) x y \\
+2 c(c l+m-1)(l x+y)+c(2 c l-1) \\
(c l+m-1)\left(l^{2} x^{2}+y^{2}\right)|-(c l+m-1) \log | 1 \\
-(c l-m)(l x+y) \mid]
\end{gathered}
$$

for all $2 c l \neq 1$ and $m \neq c l, 1-c l, 3 c l-1$, which becomes

$$
(m-c l) \log \left|V_{32}\right|-(c l+m-1) \log \left|V_{31}\right|,
$$

or equivalently, $V_{32}^{m-c l} / V_{31}^{m+c l-1}$ being $V_{31}$ and $V_{32}$, the functions defined in Theorem 2.1 (c).

If $2 c l=1$, then $V_{31}=(4 c+(2 m-1)(x+2 c y)) /(4 c), V_{32}=$ $(m-1 / 2) x+c(2+(2 m-1) y)$, and the first integral of the system is

$$
\begin{aligned}
\frac{1}{2 c(2 m-1)^{3} V_{32}}\left[( 2 m - 1 ) ^ { 2 } \left(x^{2}\right.\right. & \left.+2 c(1+2 m) x y+4 c^{2} y^{2}\right) \\
& \left.+4 c\left(2 V_{32}-8 c\right)-16 c V_{32} \log \left|2 V_{32}\right|\right]
\end{aligned}
$$

whenever $c \neq 0$ and $m \neq 1 / 2$. However, without taking into account the constant in the denominator of the previous expression, it is defined at $c=0$ and it is not constant, but it is constant if $m=1 / 2$. So, we obtain the first integral of statement (c) of the theorem if $2 c l=1$ and $m \neq 1 / 2$.

Now we consider $c l=m$. In this case, $V_{31}=1, V_{32}=2 c+(2 c l-$ $1)\left(2 c y+x(2 c l+(2 c l-1) y)+c(2 c l-1)\left(l^{2} x^{2}+y^{2}\right)\right)$, and the first integral is

$$
\frac{1}{(2 c l-1)^{3}}\left((2 c l-1)(l x+y)-\log \left|V_{32}\right|\right),
$$

with $2 c l \neq 1$. Here also we can prove that eliminating the constant of the above expression, the remaining function is constant if $2 c l=1$; therefore, the first integral is defined whenever $c l=m$ and $2 c l \neq 1$ as appears in statement (c) of the theorem in this case. 
If $m=1-c l$, we have $V_{31}=1-(2 c l-1)(l x+y), V_{32}=2 c$ and the first integral is:

$$
\begin{aligned}
& \frac{-1}{4 c(2 c l-1)^{3}}[(2 c l-1)((-2-2 c l(-3+2 c l)) x y \\
& \left.\left.+2 c(l x+y)+c(2 c l-1)\left(l^{2} x^{2}+y^{2}\right)\right)+2 c \log \left|V_{31}\right|\right]
\end{aligned}
$$

for all $c \neq 0$ and $2 c l \neq 1$. From this previous function, we obtain the first integral of statement (c) of the theorem in the cases $m=1-c l$ and $2 c l \neq 1$. If $c=0$, the first integral is defined, and it is not constant.

If $m=3 c l-1$, then $V_{31}=1-(1-2 c l)(l x+y), V_{32}=2 c(l(x-2 c l x-$ $2 c y)+y-1)^{2}$, and the first integral is

$$
\begin{aligned}
& \frac{1}{4 c(2 c l-1)^{3}} \\
& {\left[\frac{2 c\left(c(3+4 l(2 c l-1) x)+2(2 c l-1)\left(2 c+(1-2 c l)^{2} x\right) y\right)}{V_{32}}+2 c \log \left|V_{31}\right|\right]}
\end{aligned}
$$

whenever $c \neq 0$ and $2 c l \neq 1$. The first integral obtained excluding the constant factor is defined, and it is not constant at $c=0$, but it is constant if $2 c l \neq 1$. So we get the first integral of statement (c) of the theorem in the cases $m=3 c l-1$ and $2 c l \neq 1$.

Finally, if $m=1 / 2$ and $2 c l=1$, the system (1.5) becomes

$$
\dot{x}=x+\frac{x^{2}}{4 c}+x y+c y^{2}, \quad \dot{y}=-y-\frac{x^{2}}{8 c^{2}}-\frac{x y}{2 c}-\frac{y^{2}}{2},
$$

which is a Hamiltonian system, and it coincides with system (1.7) with $a=1 /(4 c)$ and $k=1 /\left(8 c^{2}\right)$. In consequence, we have proved statement (c) of the theorem.

For system (1.6), we find the first integral associated with its integrating factor $V$, given in Theorem 2.1 (d), through the equation (2.1) obtaining

$$
\int \frac{x+a x^{2}+c y^{2}}{V} d y,
$$

and hence the first integral of statement $(\mathrm{d})$ of the theorem results.

By Theorem 2.1 (e), we know that the quadratic system (1.7) is Hamiltonian, and so its inverse integrating factor is 1 . So, from 
equation (2.1), a first integral for this system is

$$
\int\left(a x^{2}+2 m x y+c y^{2}+x\right) d y+\frac{k x^{3}}{3},
$$

which is provided in statement (e) of the theorem.

\section{APPENDIX}

On the existence of Liouvillian first integrals for the integrable saddles. Doing a linear change of coordinates and a rescaling of the independent variable, any real polynomial differential system having a weak saddle at the origin can be written as

$$
\dot{x}=y+\bar{p}(x, y), \quad \dot{y}=x+\bar{q}(x, y),
$$

where $\bar{p}$ and $\bar{q}$ are real polynomials without constant and linear terms. Doing a change of variables,

$$
x=(\bar{w}+w) / 2, \quad y=(\bar{w}-w) i / 2,
$$

and of the independent variable $T=i t$, the differential system (A.1) becomes the complex differential system

$$
\dot{w}=\bar{w}+P(w, \bar{w}), \quad \dot{\bar{w}}=-w+Q(w, \bar{w}),
$$

where $P$ and $Q$ are complex polynomials. Then the focus quantities $V_{j}$ of system (A.3) coincide with the saddle quantities $L_{j}$ of system (A.1). Due to this duality between focus quantities and saddle quantities it follows that an integrable saddle has an analytic first integral defined in a neighborhood of it. This is the reason to call such a saddle an integrable saddle. The complex change (A.2) is introduced just to show the duality of weak focus and weak saddles. We must mention that the complex system (A.3) has a local complex analytic first integral in a neighborhood of the origin; see, for more details, $[\mathbf{1 1}, \mathbf{6}]$ or $[\mathbf{7}$, Section 12]. And, going back through the change of variables, we get a local complex analytic first integral in a neighborhood of the real integrable saddle. Consequently, the real and imaginary parts of this complex analytic first integral are local analytic first integrals of the integrable saddle. 


\section{REFERENCES}

1. J.C. Artés, J. Llibre and N. Vulpe, Quadratic systems with an integrable saddle: A complete classification in the coefficient space $\mathbb{R}^{12}$, Nonlin. Anal. 75 (2012), 5416-5447.

2. N.N. Bautin, On the number of limit cycles which appear with the variation of coefficients from an equilibrium position of focus or center type, Math. Sbor. 30 (1952) 181-196, Amer. Math. Soc. Transl. 100 (1954), 1-19.

3. J. Chavarriga, H. Giacomini, J. Giné and J. Llibre, On the integrability of two-dimensional flows, J. Diff. Equat. 157 (1999), 163-182.

4. C.J. Christopher, Liouvillian first integrals of second order polynomial differential equations, Electr. J. Diff. Equat. 1999 (1999), page 7.

5. H. Dulac, Détermination et intégration d'une certaine classe d'équation différentielles ayant pour point singulier un centre, Bull. Sci. Math. 32 (1908), 230-252.

6. P.M. Elizarov, Yu.S. Ilyashenko, A.A. Shcherbakov and S.M. Voronin, Finitely generated groups of germs of one-dimensional conformal mappings, and invariants for complex singular points of analytic foliations of the complex plane, in Nonlinear Stokes phenomena, American Mathemtatical Society, Providence, RI, 1993.

7. Yu. Ilyashenko and S. Yakovenko, Lectures on analytic differential equations, Grad. Stud. Math. 86, American Mathematical Society, Providence, RI, 2008.

8. P. Joyal and C. Rousseau, Saddle quantities and applications, J. Diff. Equat. 78 (1989), 374-399.

9. W. Kapteyn, On the midpoints of integral curves of differential equations of the first degree, Nederl. Akad. Wetensch. Verslag. Afd. Natuurk. Konikl. Nederland (1911), 1446-1457 (in Dutch).

10. N New investigations on the midpoints of integrals of differential equations of the first degree, Nederl. Akad. Wetensch. Verslag. Afd. Natuurk. 20 (1912), 1354-1365, Nederl. Akad. Wetensch. Verslag. Afd. Natuurk. 21 (1912), 2733 (in Dutch).

11. J.F. Mattei and R. Moussu, Holonomie et intégrales premières, Ann. Sci. École Norm. Sup. 13 (1980), 469-523.

12. J.W. Reyn, A bibliography of the qualitative theory of quadratic systems of differential equations in the plane, Delf University of Technology, http://ta.twi.tudelft.nl/DV/Staff/J.W.Reyn.html, 1997.

13. D. Schlomiuk, J. Guckenheimer and R. Rand, Integrability of plane quadratic vector fields, Expo. Math. 8 (1990), 3-25.

14. M.F. Singer, Liouvillian first integrals of differential equations, Trans. Amer. Math. Soc. 333 (1992), 673-688.

15. Cai Sulin, The weak saddle and separatrix cycle of a quadratic system, Acta Math. Sinica 30 (1987), 553-559 (in Chinese).

16. Ye Yanqian, Qualitative theory of polynomial differential systems, Shangai Scientific \& Technical Publishers, Shangai, 1995 (in Chinese). 
17. Ye Yanqian et al., Theory of limit cycles, Transl. Math. Mono. 66, American Mathematical Society, Providence, 1984.

18. H. Zoladek, Quadratic systems with center and their perturbations, J. Diff. Equat. 109 (1994), 223-273.

Departament de Matemàtiques, Universitat Autònoma de Barcelona, 08193 Bellaterra, Barcelona, Spain

Email address: ymbolanos@gmail.com, jllibre@mat.uab.cat

Departament de Matemàtiques, Universitat Autònoma de Barcelona, 08193 Bellaterra, Barcelona, Spain

Email address: jllibre@mat.uab.cat

Departamento de Matemática, Instituto Superior Técnico, Universidade Técnica de Lisboa, Av. Rovisco Pais 1049-001, Lisboa, Portugal

Email address: cvalls@math.ist.utl.pt 